Acta Crystallographica Section B

Structural

Science

ISSN 0108-7681

Editor: Carolyn P. Brock

\title{
Disodium ditungstate
}

K. Okada, H. Morikawa, F. Marumo and S. Iwai

This electronic document was scanned from an archival copy of material deposited to accompany a paper published in an IUCr journal. In many cases the only accessible copy was a microfilm of a poor-quality original. 
Disodiun Ditungstate, $\mathrm{Na}_{2} \mathrm{~W}_{2} \mathrm{O}$

BY R. Okada, H. Morikawa, F, Marumo AND S. I wal

Research Laboratory for Engineering Materials, tokyo Institute of Technology, Heguro-ku, Tokyo, Japan

ABSTRACT

$\mathrm{Na}_{2} \mathrm{~W}_{2} \mathrm{O}, 7$, orthorhombic, Crick, $a=7.216(1), b=21.899(1)$, $c=14.726(3)$ A, $z=8, D x=5.53$. The crystals were prepared by heating an intimate mixture of $\mathrm{Na}_{2} \mathrm{WO}_{4} \cdot 2 \mathrm{H}_{2} \mathrm{O}$ and $\mathrm{H}_{2} \mathrm{WO}_{4}$ at $800^{\circ} \mathrm{C}$ ln a platinum crucible for several hours. Colourless and transparent long prismatic crystals were obtained. Wo bond lengths in octahedra split in to three groups: $1.725(9)$. $1.921(1)$ and $2.352(9) \stackrel{\circ}{A}$. Those in tetrahedral in to two groups: $1.76(1)$ and $1.807(9) \stackrel{\circ}{A}$. 
Ots erved and calculared structure factors Eo and FC are multiplied by 10 . The extinction correction is

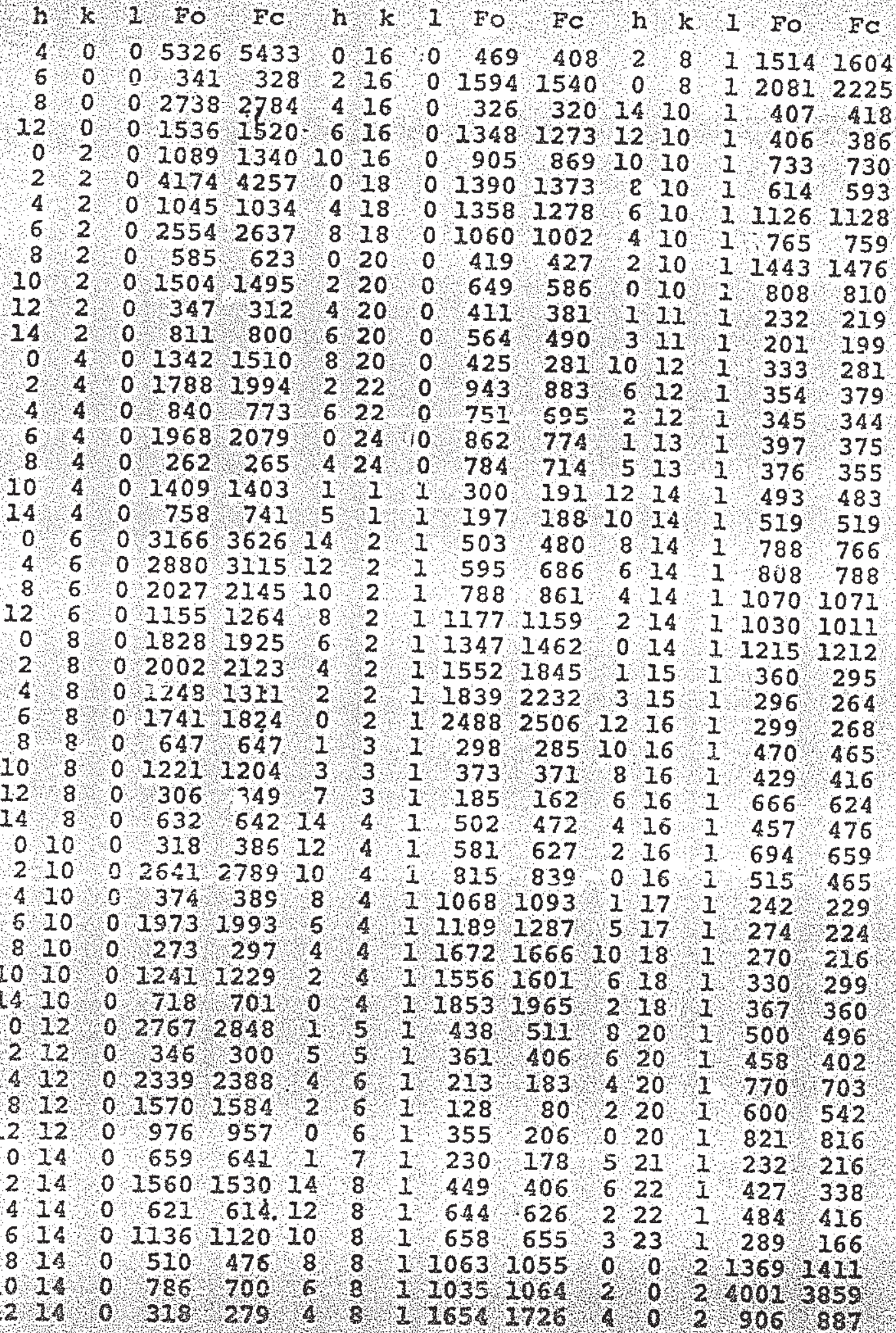


h k l Fo FC h K l FO FC h K l Fo FC 60 2 2264 2284 5 7 2 1545 1576 2 16 2 291 299 $8002 \quad 537 \quad 537 \quad 3 \quad 7 \quad 2 \quad 1552 \quad 1565 \quad 4 \quad 16 \quad 2 \quad 1042 \quad 1018$ 1000212941360 I 7 2 $19521999 \quad 6 \quad 16 \quad 2 \quad 356 \quad 260$ $12 \% 0 \quad 2 \quad 377 \quad 365 \quad 0 \quad 8 \quad 2 \quad 1644 \quad 1765 \quad 8 \quad 16 \quad 2 \quad 763 \quad 751$

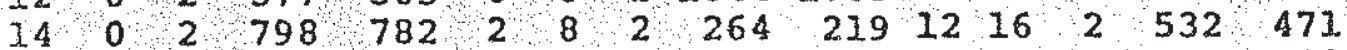
$\begin{array}{llllllllllllllll}15 & 1 & 2 & 383 & 376 & 4 & 8 & 2 & 1433 & 1535 & 11 & 17 & 2 & 326 & 320\end{array}$

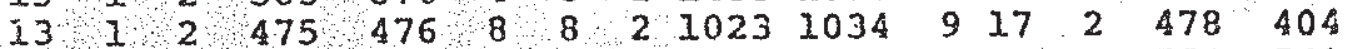

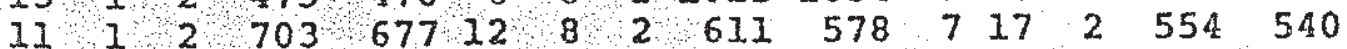
$9 I_{2} \quad 886 \quad 94013 \quad 9 \quad 2 \quad 383 \quad 420 \quad 5 \quad 17 \quad 2 \quad 550 \quad 550$

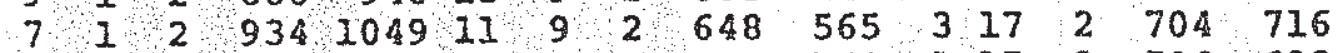
$\begin{array}{lllllllllllll}5 & 1 & 2 & 1449 & 1651 & 9 & 9 & 2 & 763 & 768 & 1 & 17 & 2\end{array}$ $311215501507 \quad 7 \quad 9 \quad 2 \quad 929 \quad 954 \quad 0 \quad 18 \quad 2 \quad 380 \quad 382$ $111223482195 \quad 5 \quad 9 \quad 2 \quad 1173 \quad 1216 \quad 2 \quad 18 \quad 2 \quad 11291106$ $022 \quad 2 \quad 24632196 \quad 3 \quad 9 \quad 2 \quad 13201390 \quad 418 \quad 2 \quad 377 \quad 364$

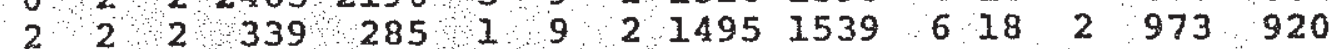
$42221514 \quad 1662 \quad 0 \quad 10 \quad 2 \quad 16421718 \quad 8 \quad 18 \quad 2 \quad 306 \quad 300$ $\begin{array}{llllllllllllll}8 & 2 & 2 & 9981085 & 2 & 10 & 2 & 241 & 100 & 10 & 18 & 2 & 708 & 639\end{array}$ $\begin{array}{llllllllllllllll}12 & 2 & 2 & 657 & 650 & 4 & 10 & 2 & 1391 & 1459 & 9 & 19 & 2 & 343 & 351\end{array}$

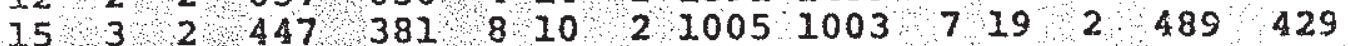

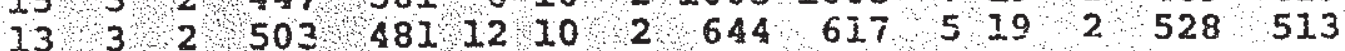

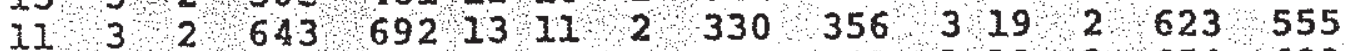

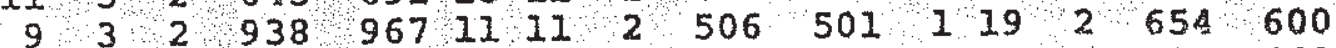

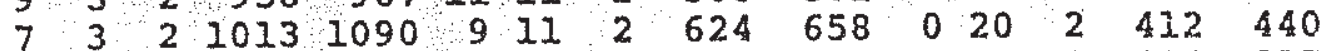

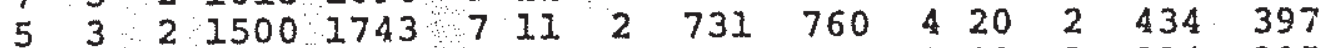

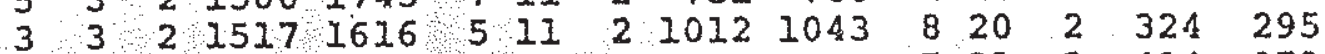
$\begin{array}{lllllllllllllll}1 & 3 & 2 & 2350 & 2298 & 3 & 11 & 2 & 1030 & 1032 & 7 & 21 & 2 & 424 & 379\end{array}$

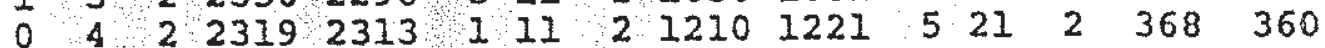

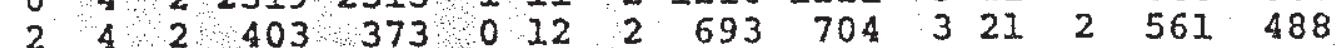
$4 \quad 4 \quad 2 \quad 20573056 \quad 212 \quad 2 \quad 17631777 \quad$ I $21 \quad 2 \quad 472 \quad 456$

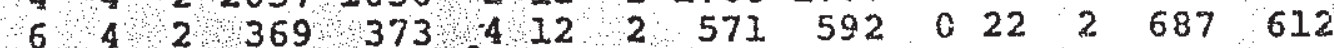
$842212981376 \quad 6 \quad 12 \quad 2 \quad 1372 \quad 1368 \quad 422 \quad 2 \quad 566 \quad 548$

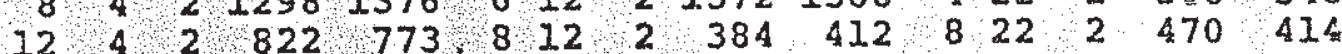

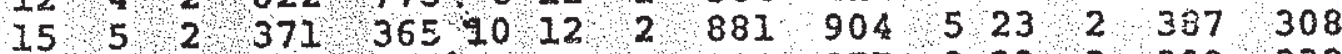
$\begin{array}{lllllllllllllll}13 & 5 & 2 & 520 & 486 & 12 & 12 & 2 & 304 & 277 & 3 & 23 & 2 & 309 & 338\end{array}$

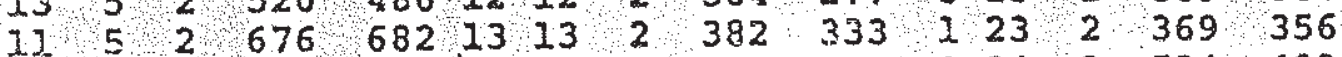
$94520962 \quad 3531113 \quad 2 \quad 482 \quad 423 \quad 2 \quad 24 \quad 2 \quad 524 \quad 499$

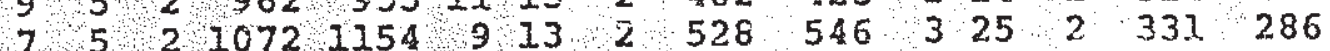

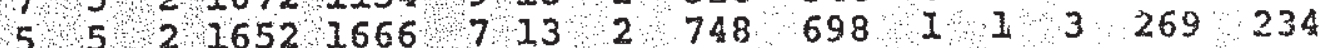
$\begin{array}{lllllllllllllll}3 & 5 & 2 & 1751 & 1788 & 5 & 13 & 2 & 788 & 772 & 3 & 1 & 3 & 264 & 293\end{array}$ 1 I. $50222042225 \quad 3 \quad 13 \quad 2 \quad 907 \quad 935 \quad 5 \quad 1 / 3 \quad 271$ 288

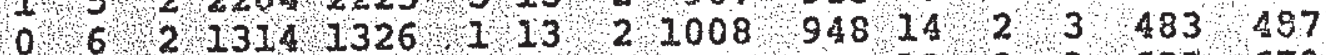
$2 \begin{array}{lllllllllllllllllll}2 & 2 & 3170 & 3199 & 0 & 14 & 2 & 769 & 721 & 12 & 2 & 3 & 635 & 679\end{array}$ $466.21120 \quad 1100 \quad 4 \quad 14 \quad 2 \quad 632 \quad 629 \quad 10022 \quad 3 \quad 797 \quad 855$

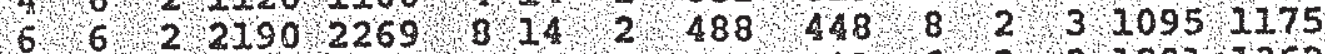

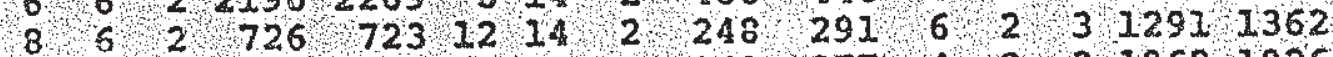

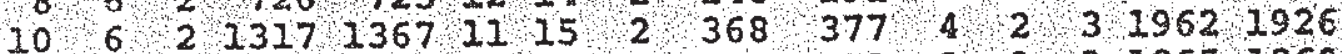
$12 \quad 6 \quad 2 \quad 426 \quad 425 \quad 9 \quad 15 \quad 2 \quad 483 \quad 479 \quad 2 \quad 2 \quad 3 \quad 18651860$

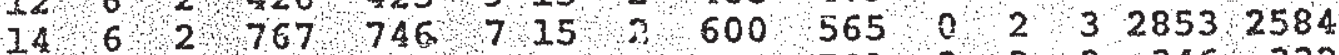
$13 \quad 7 \quad 2 \quad 463 \quad 446 \quad 5 \quad 15 \quad 2 \quad 718 \quad 721,3,3 \quad 3 \quad 346 \quad 332$ II $722 \quad 653 \quad 648 \quad 3 \quad 15 \quad 2 \quad 793 \quad 744 \quad 14 \quad 4 \quad 3 \quad 438$ 415

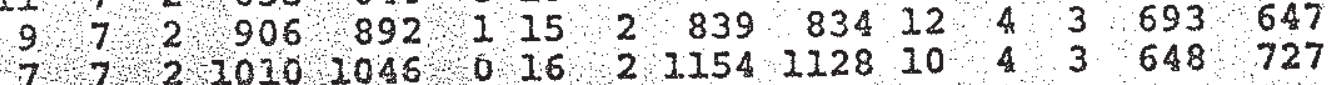




\begin{tabular}{|c|c|c|c|c|c|c|c|c|c|c|c|c|}
\hline (3) & $k$ & 1 & Fo & FC: & h & $k$ & Fo & FC & h & $\mathrm{x}$ & Fo & $\mathrm{FC}$ \\
\hline 8 & 4 & 3 & 1021 & 1094 & 42 & 20 & 381 & 348 & 2 & 6 & $\begin{array}{r}4691 \\
4657\end{array}$ & $\begin{array}{r}2682 \\
665\end{array}$ \\
\hline & 4 & $\begin{array}{l}3 \\
3\end{array}$ & & & 2 & 20 & 3 & & 4 & $\begin{array}{l}6 \\
6\end{array}$ & $\begin{array}{r}4557 \\
41784\end{array}$ & 665 \\
\hline & 4 & $\begin{array}{l}3 \\
3\end{array}$ & & $\begin{array}{l}1 \\
1\end{array}$ & $\begin{array}{l}02 \\
42\end{array}$ & 20 & & & $\begin{array}{l}6 \\
8\end{array}$ & $\begin{array}{l}6 \\
6\end{array}$ & $\begin{array}{r}1 \\
4 \quad 469\end{array}$ & 493 \\
\hline 0 & 4 & 3 & & & 02 & 22 & 566 & $\begin{array}{l}511 \\
563\end{array}$ & 10 & 6 & 41230 & $\begin{array}{r}453 \\
1287\end{array}$ \\
\hline$\frac{1}{3}$ & 5 & 3 & & & 32 & 23 & 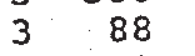 & 15 & 1 & 6 & 312 & 332 \\
\hline & 5 & 3 & 16 & 27 & 42 & 23 & 2 & 321 & 1 & & 745 & 7 \\
\hline & 5 &. & 40 & 3 & 02 & 24 & & 353 & 1. & 7 & 430 & 44 \\
\hline & 6 & & 33 & & 0 & 0 & 12971 & & 1. & 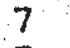 & 5 & 57 \\
\hline & 6 & & 52 & 5 & 2 & 0 & & & & 7 & 718 & 7 \\
\hline 2 & 6 & & 19 & 27 & 4 & 0 & 1 & 12 & 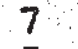 & 7 & 4 & 10 \\
\hline & 6 & 3 & 74 & 70 & 6 & 0 & 62 & 25 & 5 & 7 & 410 & 10 \\
\hline & 8 & & 43 & 522 & 8 & 0 & 6 & 8 & 3 & & 415 & \\
\hline & 8 & 3 & 43 & 509 & 10 & 0 & 15121 & 1575 & 7 & & $\begin{array}{ll}415 \\
4 & 13\end{array}$ & \\
\hline 8 & 8 & & 790 & 97 & 12 & 0 & 480 & 50 & 0 & $\begin{array}{l}8 \\
8\end{array}$ & $\begin{array}{r}4355 \\
4 \quad 985\end{array}$ & \\
\hline 6 & 8 & 3 & $\begin{array}{r}799 \\
353\end{array}$ & 8 & 14 & 0 & 3. & $\begin{array}{l}829 \\
351\end{array}$ & $\begin{array}{l}4 \\
8\end{array}$ & 8 & $\begin{array}{l}985 \\
695\end{array}$ & 8 \\
\hline 4 & 0 & 3 & $\begin{array}{l}135 \\
1.09\end{array}$ & & 15 & 1 & $\begin{array}{l}330 \\
535\end{array}$ & 530 & 12 & 8 & $\begin{array}{l}693 \\
433\end{array}$ & $\begin{array}{l}697 \\
441\end{array}$ \\
\hline 2 & 8 & 3 & & $\begin{array}{l}1175 \\
1888\end{array}$ & 31. & 1 & 687 & 715 & 13 & 9 & 477 & 35 \\
\hline 3 & 8 & 3 & 5 & 1348 . & 9 & 1 & 922 & 949 & 11 & 9 & 585 & 55 \\
\hline 3 & 2 & 3 & 3 & & 7 & 1 & 13 & 13 & 0 & 9 & 698 & 708 \\
\hline & 10 & 3 & 29 & 2 & 5 & 1 & 11 & 15 & 7 & 9 & 8 & 9. \\
\hline & 10 & 3 & 52 & 52 & 3 & 2 & 422 & 23 & 5 & 9 & 5 & $1]$ \\
\hline & 10 & 3 & 49 & 47 & 1 & 1 & 424 & 24 & 3 & 9 & 9 & 1 \\
\hline 6 & 10 & & 88 & 864 & 0 & 2 & 1 & 30 & 1 & 9 & 41 & 1 \\
\hline $\begin{array}{l}6 \\
4\end{array}$ & 10 & 3 & 709 & $7:$ & 2 & ? & 4 & 114 & 01 & 10 & 417 & 18 \\
\hline 4 & 10 & 3 & 1184 & 125 & 4 & 2 & 1 & 23 & 23 & 1 & 9 & \\
\hline 0 & 10 & 3 & & 9 & 8 & 2 & 41338 & & 2 & 18 & 16 & 1 \\
\hline 0 & 10 & 3 & 1.40 & 1458 & 12 & 2 & 4 & 738 & 6 & 1 & 8 & \\
\hline 1 & 21 & 3 & 3 & 16 & 15 & 3 & 229 & 309 & 8 & 1 & 1125 & II \\
\hline 8 & 12 & 3 & 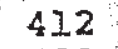 & 3 & 13 & 3 & 4 & 4 & 12 & 10 & 3 & 4 \\
\hline if & 12 & 3 & 535 & 5 & 11 & 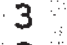 & 4 & 619 & 1.3 & 1 & 4 & 3 \\
\hline 0 & 12 & 3 & 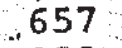 & $6 ?$ & 9 & 3 & 698 & 799 & 11 & 1 & 4 & \\
\hline 12 & 14 & 3 & & & 7 & ? & 41078 & & 9 & 11 & 4 & 5 \\
\hline 10 & 24 & 3 & & & 5 & & 41 & & 7 & . & 4 & 79 \\
\hline 8 & 14 & 3 & & & 3 & 3 & 4 & & 5 & 11 & 2 & \\
\hline 6 & 14 & 3 & 1120 & 11 & 1 & 3 & 41 & & 3 & iI. & 2 & \\
\hline 4 & 14 & 3 & 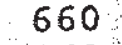 & & 0 & 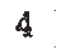 & & & 1 & 1. & 3 & \\
\hline 2 & 14 & 3 & 136 & 13 & 2 & 4 & 35 & & 0 & $1:$ & 8 & \\
\hline 0 & 24 & - & 72 & & 4 & 4 & 42163 & 21 & 2 & 12 & 2041 & \\
\hline 12 & 16 & 3 & 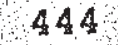 & & 6 & $x$ & 4 & & 4 & 12 & 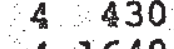 & \\
\hline 10 & 16 & 3 & 21 & 2 & 8 & 4 & 411 & 12 & 6 & 1 & 1649 & \\
\hline 8 & 16 & 3 & -7 & & 12 & 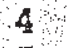 & 70 & 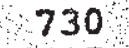 & 8 & 1 & 4 & \\
\hline 6 & 16 & 3 & & & 15 & 5 & 4 & 2 & 10 & 1 & 410 & \\
\hline 4 & 16 & 3 & & & 13 & 5 & 4 & & 12 & 1 & 263 & \\
\hline 2 & 16 & 3 & $\varepsilon_{1}$ & & 11 & 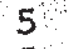 & 4 & 5 & 13 & 1 & 4 & \\
\hline 0 & 16 & 3 & & & 9 & 5 & 4 & & 11 & 1 & 4. & 4 \\
\hline 8 & 18 & 3 & & & 7 & & 943 & & 9 & $\begin{array}{l}13 \\
13\end{array}$ & $\begin{array}{l}566 \\
665\end{array}$ & $\frac{5}{6}$ \\
\hline 4 & 18 & $\because 2$ & & & 5 & 5 & 4 & & 5 & 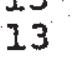 & 810 & \\
\hline & 2 & 3 & & & 0 & & $\begin{array}{l}49 \\
\quad \quad 879\end{array}$ & $102 !$ & 1 & 13 & 995 & \\
\hline
\end{tabular}




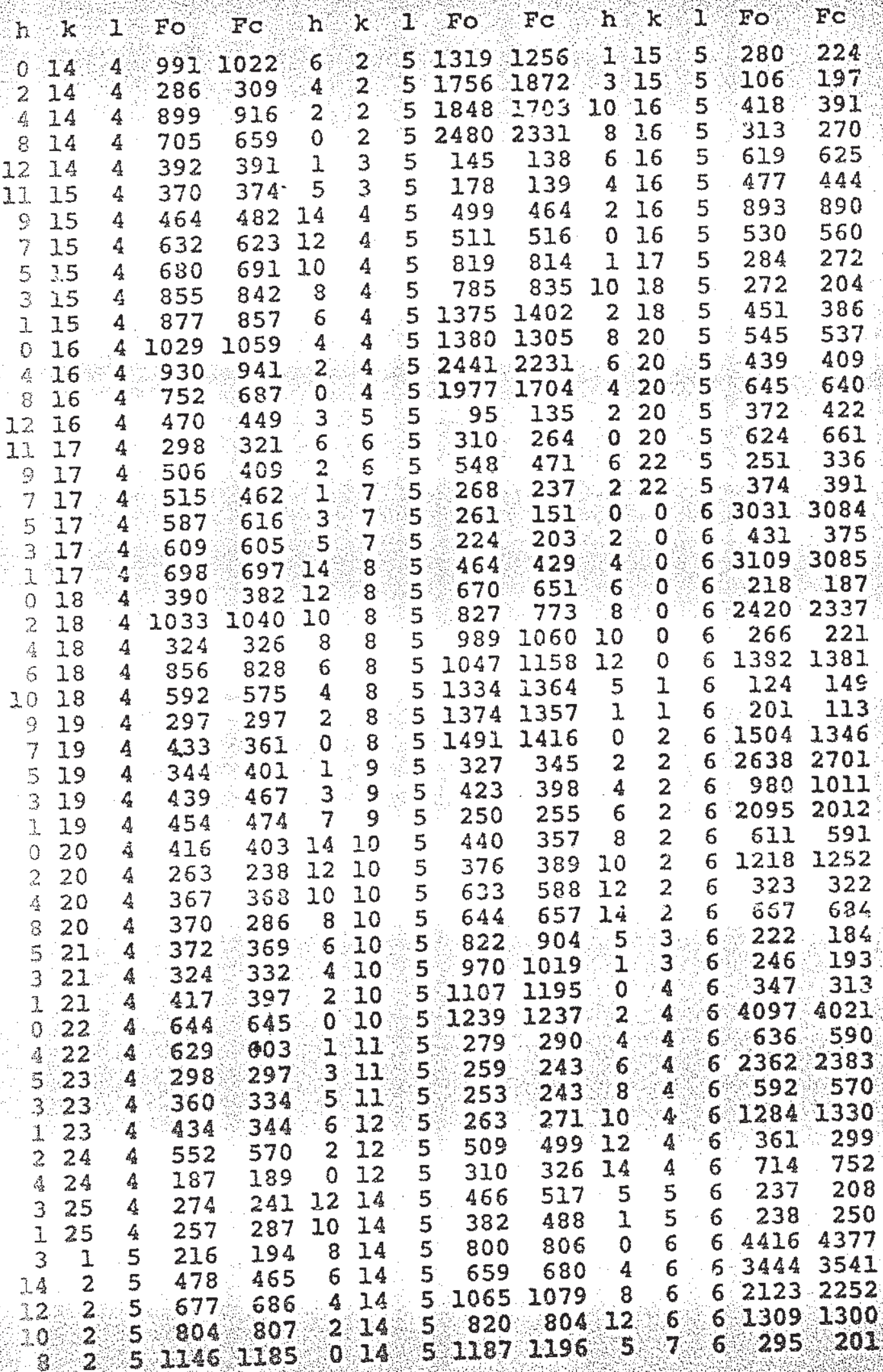




\begin{tabular}{|c|c|c|c|c|c|c|c|c|c|c|c|c|c|c|}
\hline h & $k$ & 1 & FO & FC & h & k & 1 & Fo & $\mathrm{FC}$ & h & $k$ & 1 & Eo & FC \\
\hline $\begin{array}{l}1 \\
0\end{array}$ & $\begin{array}{l}7 \\
8\end{array}$ & $\frac{6}{6}$ & $\begin{array}{l}211 \\
654\end{array}$ & 237 & 1 & 3 & 7 & $\begin{array}{l}360 \\
291\end{array}$ & $\begin{array}{l}290 \\
312\end{array}$ & $\begin{array}{l}8 \\
6\end{array}$ & $\begin{array}{l}16 \\
16\end{array}$ & $\begin{array}{l}7 \\
7\end{array}$ & $\begin{array}{l}310 \\
616\end{array}$ & $\begin{array}{l}287 \\
590\end{array}$ \\
\hline 2 & 8 & 6 & 2572 & 2642 & 14 & 4 & & 424 & $\triangle A \triangle$ & 4 & 16 & 7 & 389 & 435 \\
\hline 4 & 8 & 6 & 694 & 704 & 2. & 4 & 7 & 492 & 520 & 2 & 16 & 7 & 760 & 800 \\
\hline 6 & 8 & 6 & 1567 & 1721 & 10 & 4 & 7 & 787 & 770 & 0 & 16 & 7 & 526 & 526 \\
\hline 8 & 8 & 6 & 642 & 608 & 8 & 4 & 7 & 899 & 851 & 3 & 17 & 7 & 8 & 243 \\
\hline 10 & 8 & 6 & 1019 & 997 & 6 & A & 7 & 1309 & 1271 & 10 & 18 & 7 & 293 & 210 \\
\hline$\alpha$ & 8 & 6 & 397 & 3. & 4 & 4 & 7 & 1260 & 1298 & 6 & 18 & 7 & 348 & 307 \\
\hline 14 & 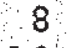 & 6 & 637 & 57 & 2 & 4 & 7 & 2027 & 18 & 2 & 18 & 7 & 351 & 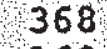 \\
\hline 0 & 10 & 6 & 644 & 625 & 0 & 4 & 7 & 1845 & 16 & 1 & 19 & 7 & 206 & 168 \\
\hline- & 10 & 6 & 2067 & 205 & 3 & 5 & 7 & 336 & 298 & 8 & 20 & 7 & 495 & 502 \\
\hline 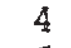 & 10 & 6 & 462 & 41 & 7 & 5 & 7 & 151 & 182 & 6 & 20 & & 396 & 403 \\
\hline 6 & 10 & 6 & 1581 & 167 & 6 & 6 & 7 & 148 & 162 & 4 & 20 & 7 & 591 & 612 \\
\hline$\varepsilon$ & 20 & 6 & 267 & 25 & 4 & 6 & 7 & 159 & 156 & 2 & 20 & 7 & 470 & 435 \\
\hline ( & 10 & 6 & 2133 & 120 & 2 & 6 & 7 & 232 & 2 & 0 & 20 & & 65 & 2 \\
\hline 0 & 10 & 6 & 631 & 62 & 0 & - & 7 & 257 & & 6 & 22 & 7 & 269 & 308 \\
\hline 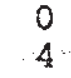 & 12 & 6 & 200 & 218 & 1 & 7 & 7 & 437 & 396 & 2 & 22 & 7 & 324 & 349 \\
\hline & 12 & 6 & 1936 & 201 & 3 & 7 & 7 & 38 & 3 & 0 & 2 & & 3 & 27 \\
\hline & 12 & 6 & 1541 & 152 & 5 & 7 & 7 & 263 & 274 & 2 & 24 & 7 & 290 & 247 \\
\hline & 12 & 6 & 99 & 943 & 7 & 7 & 7 & 268 & 231 & 0 & 0 & 8 & 1652 & 1592 \\
\hline & 1 & & 784 & 840 & 14 & 8 & 7 & 3 & 4 & 2 & 0 & 8 & 2858 & 2830 \\
\hline 4 & 1 & 6 & 1254 & 1139 & 12 & 8 & 7 & $\begin{array}{l}646 \\
739\end{array}$ & 9 & $\begin{array}{l}4 \\
6\end{array}$ & $\begin{array}{l}0 \\
0\end{array}$ & 8 & $\begin{array}{l}5 \\
2\end{array}$ & $\begin{array}{l}20 \\
88\end{array}$ \\
\hline 6 & 14 & 6 & 995 & $\begin{array}{l}691 \\
976\end{array}$ & 8 & 8 & 7 & 986 & 1007 & $\begin{array}{l}6 \\
8\end{array}$ & $\begin{array}{l}0 \\
0\end{array}$ & $\begin{array}{l}8 \\
8\end{array}$ & $\begin{array}{r}2082 \\
883\end{array}$ & $\begin{array}{r}2088 \\
860\end{array}$ \\
\hline 8 & 24 & 6 & 403 & 438 & 6 & 8 & 7 & 1017 & 1070 & 10 & 0 & 2 & 1300 & 1288 \\
\hline & 14 & 6 & 71 & 672 & 4 & 8 & 7 & 13 & 13 & 12 & 0 & 8 & 561 & 497 \\
\hline 2 & 1.4 & 6 & 291 & 26 & 2 & 8 & 7 & 1412 & 1296 & 14 & 0 & 8 & 725 & 709 \\
\hline 2 & 16 & 6 & 1454 & 150 & 0 & 0 & 7 & 1599 & 1470 & 15 & $I$ & 8 & 263 & 320 \\
\hline 6 & 16 & 6 & 1178 & 1174 & 1 & 9 & 7 & 251 & 253 & 13 & 1. & 8 & 478 & 486 \\
\hline 10 & 16 & 6 & 852 & 782 & 3 & 0 & & 4 & 415 & 11 & I & 8 & 624 & 650 \\
\hline 0 & 38 & 6 & 1425 & 1477 & 12 & 10 & 7 & 393 & 365 & 9 & 1 & 8 & 905 & 832 \\
\hline 4 & 38 & 6 & 1298 & 1294 & 10 & 10 & 7 & 5 & 616 & 7 & 1 & 8 & 1245 & 1233 \\
\hline 8 & 18 & 6 & 1002 & 923 & 8 & $\perp$ & & & 593 & 5 & 1 & 8 & 1282 & 1271 \\
\hline 0 & 20 & 6 & 47 & 424 & 6 & 10 & 7 & 845 & $S 44$ & 3 & $I$ & 3 & 1354 & 1877 \\
\hline 2 & 20 & 6 & 66 & 678 & 4 & 10 & 7 & 820 & 7 & 1 & 1 & 8 & & 1795 \\
\hline 4 & 20 & 6 & 33 & 38 & 2 & 1 & & 12 & 1216 & 0 & 2 & 8 & 2008 & 1909 \\
\hline 6 & 20 & 6 & $5 t$ & 54 & 0 & 10 & 7 & 1.047 & 972 & 2 & 2 & 8 & 332 & 295 \\
\hline 8 & 20 & 6 & 2 & 287 & 1 & 11 : & 7 & 143 & 225 & 4 & 2 & 8 & 1571 & 1630 \\
\hline 2 & 22 & 6 & 768 & 768 & 3 & 11 & 7 & 164 & 2 & 5 & 2 & 8 & 287 & 252 \\
\hline 0 & 22 & 6 & 706 & 676 & 2 & 12 & 7 & 390 & 424 & 8 & 2 & 8 & 1092 & 1081 \\
\hline 0 & 24 & 6 & & 70 & 9 & 13 & 7 & 204 & 220 & 10 & 2 & 8 & 179 & 156 \\
\hline 4 & 24 & 6 & 638 & 644 & 3 & 13 & 7 & 2 & 332 & 12 & 2 & 8 & 642 & 611 \\
\hline 1 & 1 & 7 & 407 & 341 & 7 & 13 & 7 & 26 & 246 & 13 & 3 & 8 & 560 & 531 \\
\hline 5 & 1 & 7 & & & 12 & 14 & 7 & 433 & 469 & 11 & 3 & 8 & $7=$ & 6 \\
\hline 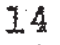 & 2 & 7 & & 43 & 10 & 14 & 7 & 445 & 489 & 9 & 3 & 8 & & 891 \\
\hline & 2 & 7. & 76 & 670 & 8 & 14 & 7 & 767. & 72 & 7 & 3 & 8 & 1386 & 1299 \\
\hline 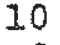 & 2 & 7 & 92 & & 6 & 14 & 7 & 686 & 705 & 5 & 3 & 8 & 1262 & 13 \\
\hline 8 & 2 & 7 & & 11 & 4 & 14 & 7 & 988 & 976 & 3 & 3 & 8 & 2032 & 2009 \\
\hline 6 & 2 & 7 & 1180 & 11 & 2 & 1.4 & 7 & 800 & 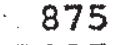 & 1 & 3 & 8 & 2110 & 1961 \\
\hline A & 2 & 7 & 1742 & 18 & 0 & 14 & 7 & 1031 & 1095 & 0 & 4 & 8 & 2044 & 1879 \\
\hline 2 & 2 & 7 & 1559 & & 3 & 15 & 7 & & 264 & 4 & 4 & & & 1523 \\
\hline & 2 & 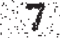 & 2355 & & & & 7 & & & & 4 & & 1010 & 100 \\
\hline
\end{tabular}




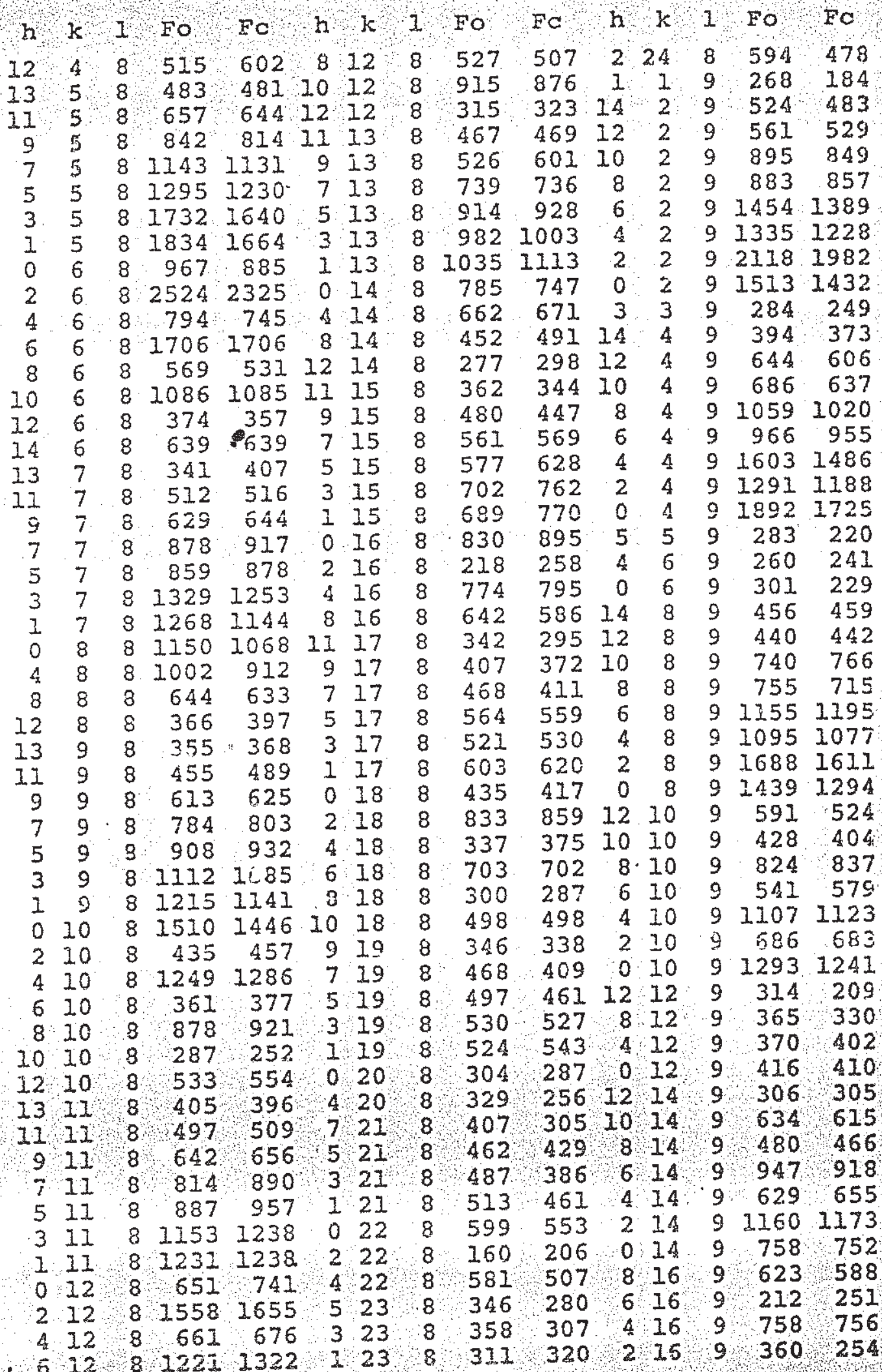




\begin{tabular}{|c|c|c|c|c|c|c|c|c|c|c|c|c|c|}
\hline$k$ & 1 & Fo & Fo & h & k & 1 & Fo & $F C$ & $\mathrm{~h}$ & $k$ & 1 & Fo & EC \\
\hline 16 & 9 & 845 & 817 & 2 & 6 & 10 & 2811 & 2730 & 6 & 14 & 10 & 223 & 210 \\
\hline $7 \rightarrow$ & 0 & & & 4 & 6 & 10 & 766 & 731 & 8 & I4 & 10 & 500 & 450 \\
\hline 18 & 9 & 39 & & 6 & 6 & 10 & 2006 & 2015 & 12 & 14 & 10 & 380 & \\
\hline 18 & 9 & 43 & 4 & 8 & 6 & 10 & 438 & 442 & 11 & 15 & 10 & & 303 \\
\hline 20 & 9 & 34 & & 10 & 6 & 10 & 1280 & 1248 & 9 & 15 & 7 & & 376 \\
\hline 20 & 9 & 637 & 619 & 12 & 6 & 10 & 355 & 278 & 7 & 15 & 0 & 1 & 45 \\
\hline 20 & 9 & 374 & 325 & 34 & 6 & 10 & 779 & 701 & 5 & 15 & 10 & 539 & 58 \\
\hline 20 & 9 & 81 & & 13 & 7 & 10 & 357. & 352 & 3 & 15 & 1.0 & 560 & 78 \\
\hline 20 & 9 & 40 & 8 & 11 & 7 & 10 & 499 & 485 & 1 & IE & 1 & 600 & 43 \\
\hline $\begin{array}{l}22 \\
22\end{array}$ & S & 42 & 407 & 9 & 7 & 10 & 667 & 648 & 0 & 16 & 10 & 1056 & 1061 \\
\hline $\begin{array}{l}22 \\
24\end{array}$ & 9 & 478 & 439 & 7 & 7 & 10 & 752 & 773 & 4 & 16 & 10 & 970 & 62 \\
\hline $\begin{array}{r}24 \\
0\end{array}$ & 9 & 328 & 301 & 5 & 7 & 10 & 1043 & 10 & 8 & 16 & 10 & 745 & 725 \\
\hline $\begin{array}{l}0 \\
0\end{array}$ & 10 & 1072 & 984 & 3 & 7 & 10 & 1187 & 1083 & 9 & 17 & 10 & 385 & 316 \\
\hline $\begin{array}{l}0 \\
0\end{array}$ & 10 & 301 & 2934 & 1 & 7 & 10 & 1394 & 1255 & 7 & 17 & 10 & 401 & 408 \\
\hline $\begin{array}{l}0 \\
0\end{array}$ & 10 & 621 & 634 & 0. & 8 & 10 & 1617 & 1525 & 5 & 17 & 10 & 474 & 427 \\
\hline $\begin{array}{l}0 \\
0\end{array}$ & 10 & 1957 & 1966 & 2 & 8 & 10 & 493 & 460 & 3 & 17 & 10 & 532 & 531 \\
\hline $\begin{array}{l}0 \\
0\end{array}$ & 10 & 310 & 315 & 4 & 8 & 10 & 1424 & 1396 & 1. & 17 & 10 & 496 & 23 \\
\hline 0 & 10 & $1240^{\circ}$ & 1208 & 6 & 8 & 10 & 109 & 216 & 2 & 18 & 10 & 1065 & 1030 \\
\hline 0 & 10 & $740^{\circ}$ & 730 & 8 & 8 & 10 & 1029 & 1019 & 4 & 18 & 10 & 164 & 212 \\
\hline 1 & 10 & 425 & 39 & 12 & 8 & 10 & 650 & 592 & 6 & 18 & 10 & 996 & 5 \\
\hline $\begin{array}{l}1 \\
I\end{array}$ & 10 & 554 & 534 & 13 & 9 & 10 & 35 & 32 & 8. & 18 & 10 & 3 & 8 \\
\hline $\begin{array}{l}1 \\
I\end{array}$ & 10 & 752 & 728 & 11 & 9 & 10 & 468 & 438 & 9 & 19 & 10 & 310 & 66 \\
\hline $\begin{array}{l}1 \\
1\end{array}$ & 10 & 871 & 867 & 9 & 9 & 10 & 570 & 56 & 7 & 19 & 10 & 321 & 309 \\
\hline$\frac{1}{1}$ & 10 & 1175 & 1.138 & 7 & 9. & 10 & 712 & 72 & 5 & 19 & 10 & 429 & 6 \\
\hline$\frac{1}{1}$ & 10 & 126 & 123 & 5 & 9 & 10 & 862 & 847 & 3 & 19 & 10 & 461 & 96 \\
\hline$\frac{1}{2}$ & 10 & 1468 & Is & 3 & 9 & 10 & 1026 & 1002 & 1 & 19 & 10 & 14 & 9 \\
\hline $\begin{array}{l}2 \\
2\end{array}$ & 10 & 1709 & 163 & 1 & 9 & 10 & 11 & 10 & 0 & 20 & 10 & 424 & $I$ \\
\hline 2 & 10 & $x \geq 2$ & 30 & 0 & 10 & 10 & 1604 & 15 & 4 & 20 & 10 & 439 & 3 \\
\hline$?$ & 10 & 1550 & 1443 & 4 & 10 & 10 & 1323 & 1348 & 8 & 20 & 10 & 357 & 2 \\
\hline 2 & 10 & 198 & 222 & 8 & 10 & 10 & 908 & 9 & 7 & 21 & 10 & 271 & 4 \\
\hline 2 & 10 & 1092 & 1044 & 32 & 10 & 10 & 610 & 600 & 5 & 21 & 10 & 05 & 71 \\
\hline 2 & 10 & 698 & 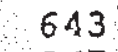 & 13 & 12 & 10 & 257 & 281 & 3. & 21 & 0 & 6 & 8 \\
\hline $\begin{array}{l}3 \\
3\end{array}$ & 10 & 38 & 377 & 11 & 11 & 10 & 332 & 3 & 1 & 21 & 10 & 5 & 5 \\
\hline 3 & 10 & 587 & 5 & 9 & 11 & 10 & 500 & 50 & 0 & 22 & 10 & 6 & I. \\
\hline 3 & 10 & 705 & 75 & 7 & 11 & 10 & 624 & 593 & 4 & 22 & 10 & 32 & 3 \\
\hline $\begin{array}{l}3 \\
3\end{array}$ & 10 & 873 & 86 & 5 & 11 & 10 & 736 & 77 & 3 & 23 & 10 & 175 & 254 \\
\hline 3 & 10 & 1219 & 115 & 3 & 11 & 10 & 764 & 79 & 1 & 23 & 10 & & 77 \\
\hline 3 & 10 & 1320 & 12 & 1 & 11 & 10 & 908 & 90 & 2 & 24 & & 5 & 493 \\
\hline 3 & 10 & 15 & 1 & 0 & 12 & 10 & 532 & & 1 & 1 & 11 & 139 & 7 \\
\hline 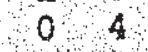 & 10 & 1999 & 18 & 2 & 12 & 10 & 1513 & 1596 & 14 & 2 & 11 & 0 & 9 \\
\hline 6 & 10 & 1858 & 17 & 4 & 12 & 10 & 418 & 417 & 12 & 2 & 11 & 583 & 5 \\
\hline 4 & 10 & 23 & 1 & 6 & 12 & 10 & 1162 & 1236 & 10 & 2 & 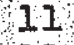 & 814 & \\
\hline 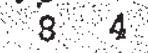 & 10 & 1353 & 1292 & 8 & 12 & 10 & 290 & 278 & 8 & 2 & 11 & 873 & \\
\hline 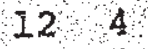 & 10 & 806 & 750 & 10 & 12 & 10 & 8 & 0 & 6 & 2 & 11 & 1182 & \\
\hline & 10 & 40 & 378 & 11 & 13 & 10 & 451 & & 4 & & 11 & 12 & \\
\hline 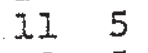 & 10 & 601 & 509 & 9. & 13 & 10 & 410 & 42 & 2 & 2 & 11 & 1653 & \\
\hline 5 & 10 & 671 & 60 & 7 & 13 & 10 & 53 & 54 & 0 & 2 & 11. & 2544 & 1483 \\
\hline 7 & 10 & 91 & 0 & 5 & 13 & 10 & 557 & 60 & I & 3 & & & 70 \\
\hline 5 & 10 & 1.169 & 10 & 3 & 13 & 10 & 702 & 725 & 3 & 3 & & & \\
\hline 5 & 10 & 1326 & & 1 & 13 & 10 & 697 & 737 & 14 & 4. & $I I$ & 450 & 400 \\
\hline 5 & 10 & 15 & & 0 & 14 & 20 & 68 & 739 & 12 & 4 & 31 & & \\
\hline 6 & 1 & & & 2 & 14 & & 560 & 629 & 10 & 4 & & & \\
\hline
\end{tabular}




\begin{tabular}{|c|c|c|c|c|c|c|c|c|c|c|c|c|c|}
\hline k & $i$. & Fo & FC & $\mathrm{h}$ & $k$ & 1 & 0 & FC & $\mathrm{h}$ & & & Fo & \\
\hline 4 & 71 & 875 & 867 & 4 & 0 & 12 & 051 & 3082 & $\begin{array}{r}8 \\
10\end{array}$ & 14 & 12 & 18 & \\
\hline 4 & & & & 8 & & 12 & & & 10 & & & & \\
\hline 4 & 11 & 72 & 1210 & 12 & 0 & 12 & 9 & & 7 & & 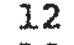 & & \\
\hline 4 & T & & & 7 & & & & & 2 & & & & \\
\hline 4 & & & & & & & & & 6 & & 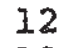 & & \\
\hline 5 & 11 & & & 3 & 7 & & & & 10 & & 12 & & \\
\hline 7 & & & 8 & 1 & 1 & 1 & & & 0 & 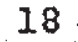 & 12 & 1166 & \\
\hline 8 & & & $5:$ & 0 & & 12 & & & 4 & & 12 & & \\
\hline 8 & 7 & 5 & & 2 & & 12 & 2532 & 25 & 8 & & 12 & & \\
\hline 8 & 1. & & & 4 & 2 & 12 & & & 0 & & 12 & & \\
\hline 8 & & & & 6 & & 12 & 18 & & 2 & 0 & 12 & & \\
\hline 8 & 1. & 135 & 13 & 8 & & 12 & & & 4 & & & & \\
\hline 8 & 13 & & & 10 & & 12 & & & 6 & 0 & 12 & & \\
\hline 8 & 7 & & & 12 & & 12 & & & 2 & 2 & 12 & & \\
\hline 9 & 7 & & & 14 & & 12 & & 6 & 1 & 1 & 13 & & \\
\hline 9 & 1 & 1 & & 1. & & 1 & & 75 & 14 & 4 & 13 & & \\
\hline 10 & 1 & & & & & 12 & 29 & & 2 & 2 & 13 & & \\
\hline & & & & 3 & 3 & 12 & & & 10 & 2 & 13 & & \\
\hline 10 & 1 & 5 & & 0 & & 12 & 11 & 10 & 8 & 2 & 13 & & \\
\hline 10 & 1 & 88 & & & & 12 & 20 & & 6 & 2 & 13 & & \\
\hline 70 & 9 & & & 4 & 4 & 12 & & & 4 & 2 & 13 & & \\
\hline 3 & & 10 & 10 & & & 12 & 17 & 1.7 & 2 & 2 & 13 & & \\
\hline 10 & 1 & & & & & 12 & & & 0 & 2 & 13 & & \\
\hline 7. & & & & 1 & 4 & 12 & 12 & & 1 & 3 & 13 & & \\
\hline 13 & & & & 1 & & 12 & & & 3 & 3 & 1 & & \\
\hline 1: & & & & & & 12 & & & 7 & 3 & 1 & & \\
\hline 7 & & & & 0 & & 12 & 25 & 25 & 14 & 4 & 13 & & \\
\hline 1 & & & & 4 & & 12 & 22 & & 12 & 4 & 13 & & \\
\hline 1 & & & & & & 12 & & & 10 & 4 & 3 & & \\
\hline 1. & & & & 12 & 6 & 12 & & & 8 & 4 & & & \\
\hline & & 8 & & & & 12 & & & 6 & 4 & 13 & & \\
\hline 1. & & & & & & 12 & & & 4 & $A$ & 13 & & \\
\hline 1. & & & & 2 & & 12 & 1.4 & & 2 & 4 & 13 & & \\
\hline I. & & & & 4 & & 12 & & & 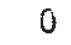 & 4 & 23 & & \\
\hline I & & & & 6 & & 12 & 12 & & & 5 & 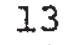 & & \\
\hline 16 & & & & & & 12 & & & 2 & 6 & & & \\
\hline & & & & 10 & 8 & 12 & & & 7 & 7 & 1 & 1 & \\
\hline 1 & & & & 3 & 9 & 1 & & & 12 & 8 & & & \\
\hline It & & & & 0 & 10 & 12 & & & lu & 8 & 13 & & \\
\hline & & & & $?$ & 70 & 12 & 39 & 19 & 0 & 8 & 13 & & \\
\hline 1 & & & & $A$ & 10 & 12 & & & . & & & & \\
\hline 18 & 1. & & & 6 & 1 & 12 & 14 & & 4 & 3 & 13 & & \\
\hline 28 & & & & 10 & 7 & 12 & 10 & & 2 & B & 13 & & \\
\hline 2 & & & & 2 & & 12 & & & 8 & 8 & 13 & & \\
\hline 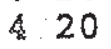 & & & & 0 & 12 & 12 & 21 & & 1 & 9 & & & \\
\hline a & 1 & & & $z$ & . & 12 & & & 5 & 9 & 1 & & \\
\hline 2 & & & & & & 12 & 12 & & 12 & 10 & $i$ & & \\
\hline $2:$ & & & & 12 & 1 & 12 & & & 10 & 10 & & & \\
\hline 22 & & & & 0 & 14 & 12 & & & 8 & 10 & 13 & & \\
\hline 22 & & & & & & 1 & & 11 & 6 & 30 & $I$ & & \\
\hline & & & & & & 7 & & & 4 & 10 & 13 & & \\
\hline & & & & & & & & & -3 & 10 & 3 & 874 & \\
\hline
\end{tabular}




\begin{tabular}{|c|c|c|c|c|c|c|c|c|c|c|c|c|c|}
\hline k & $I$ & Fo & FC & & $\mathrm{k}$ & 1 & Fo & FC & $\mathbf{h}$ & $k$ & 7 & Fo & \\
\hline 70 & 13 & 842 & 838 & 1 & 3 & i4 & 1342 & 1378 & 8 & 12 & 14 & 433 & 10 \\
\hline & 13 & & & & & 74 & 6 & 1283 & 10 & 12 & & & \\
\hline 1 & 13 & & & 2 & & 14 & 538 & 511 & 11 & 13 & $\Delta$ & & \\
\hline 12 & 13 & 25 & & 4 & 4 & 14 & 1240 & 1110 & 9 & 13 & & & \\
\hline 13 & 13 & & & 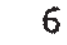 & & 14 & 304 & 3 & 7 & 3 & & & \\
\hline & 13 & & & & & 1 & 7 & & 5 & 13 & & & \\
\hline & 13 & 20 & 1 & 12 & & 14 & & & 3 & 13 & & & \\
\hline & 13 & & & 13 & & 14 & & & 1 & 13 & & & \\
\hline 14 & 13 & & & 11 & & 1 & & 5 & 0 & 14 & & & \\
\hline $1-$ & 13 & $2=6$ & & & & 1 & & 698 & 4 & 14 & & & \\
\hline$\because A$ & 13 & & & 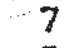 & & 14 & & 829 & 9 & 15 & & & \\
\hline & 13 & & & 5 & & 14 & 10 & 10 & 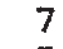 & 15 & & & \\
\hline d. & 13 & 89 & & 7 & & 14 & 1152 & 11 & 5 & 15 & & & \\
\hline & 13 & 40 & & 1 & $=$ & 14 & 1342 & 1304 & 3 & 15 & & & \\
\hline & 23 & & & 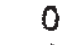 & & 14 & 1 & & 1 & 15 & & & \\
\hline 16 & 13 & 48 & 48 & 2 & & 14 & 1642 & 16 & 0 & 16 & & & \\
\hline & 1 & 362. & & 4 & 6 & 14 & 904 & & 4 & 16 & & 0 & \\
\hline & 13 & . & & 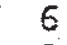 & & 14 & $127 \AA$ & 1331 & 8 & 16 & & & \\
\hline & 13 & 4 & 373 & 0 & 6 & 14 & 590 & 659 & 10 & 16 & & & \\
\hline 1 & 13 & 1 & & 10 & 6 & 14 & 90 & 894 & 9 & 17 & & & \\
\hline & 2. & & & 12 & & & & & 7 & 17 & & & \\
\hline 2 & 13 & 3 & & 13 & & & 38 & & 5 & 17 & & & \\
\hline & 13 & & & II & 7 & 1 & 52 & 50 & 3 & 17 & & & \\
\hline 2 & 13 & & & & & & & 6 & & 17 & & & \\
\hline 20 & 13 & 3 & & 7 & 7 & 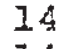 & 7 & 7 & 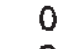 & 18 & & 45 & \\
\hline & 1 & & & 5 & & $\Delta$ & & & 2 & 18 & & 73 & \\
\hline & 33 & 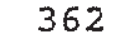 & & 3 & 7 & 14 & 1 & & 4 & 18 & & 3. & \\
\hline 0 & 24 & $719^{\circ}$ & 5 & $I$ & 7 & & 12 & 1 & 6 & 8 & & 6 & \\
\hline & 7 & 15 & & 0 & & & & & 8 & 8 & & & \\
\hline 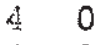 & 14 & & & 4 & & 1 & 8 & 8 & 7 & 9 & & & \\
\hline 0 & 14 & 1459 & 14 & 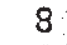 & & $y$ & & 57 & 5 & 9 & & 4 & \\
\hline & 14 & & & II & & & & & 3 & 19 & & & \\
\hline & 70 & & & 11 & & $\dot{-}$ & 4 & & 1 & 19 & & & \\
\hline 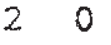 & 14 & & & 9 & & 1 & 56 & 5 & 0 & 20 & & & \\
\hline , & 14 & & & 7 & & & & & 4 & 20 & & & \\
\hline & 1 & & & 5 & & & & & 5 & $2 I$ & & & \\
\hline 1 & 14 & & & 3 & 9 & 14 & & & 3 & 21 & & & \\
\hline 7 & 14 & & & $\alpha$ & 9 & 14 & 10 & 70 & 1 & 21 & & & \\
\hline & 1 & & & 0 & 10 & & & & 0 & 22 & & & \\
\hline 1 & 34 & & & 4 & 1 & 1 & & & 4 & 22 & & & \\
\hline 7 & 14 & & & 8 & 1 & 1 & 6 & 63 & 1 & 23 & & 2 & \\
\hline 1 & 14 & & & 12 & 1 & 1 & & 407 & 12 & 2 & & & \\
\hline 2 & 1 & & & 11 & 1 & 1 & & & 10 & 2 & & & \\
\hline 2 & IA & & & 9 & 2 & 1 & & & 8 & $?$ & & & \\
\hline 2 & 14 & & & 7 & 1 & 1 & 5 & 58 & 6 & 2 & & 9 & \\
\hline$\lambda$ & 1 & & & 5 & 11 & 1 & 76 & 77 & 4 & 2 & & & \\
\hline 3 & 1 & & & 3 & 1. & 1 & $7 \%$ & 77 & 2 & 2 & & & \\
\hline 3 & 1 & & & 9 & 1. & 7 & 86 & 882 & 0 & 2 & & 1180 & \\
\hline & 14 & & & 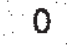 & - & 7 & & 579 & 12 & 4 & & 478 & \\
\hline 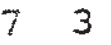 & 1 & & & $\sim$ & $I$ & 1 & 11 & 1116 & I0 & 4 & & & \\
\hline 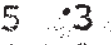 & 7 & & & 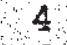 & $I$ & 7 & & 5 & 8 & 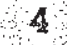 & & & \\
\hline 3 & 1 & & & 6 & 1 & & & & 6 & 4 & & 76 & \\
\hline
\end{tabular}




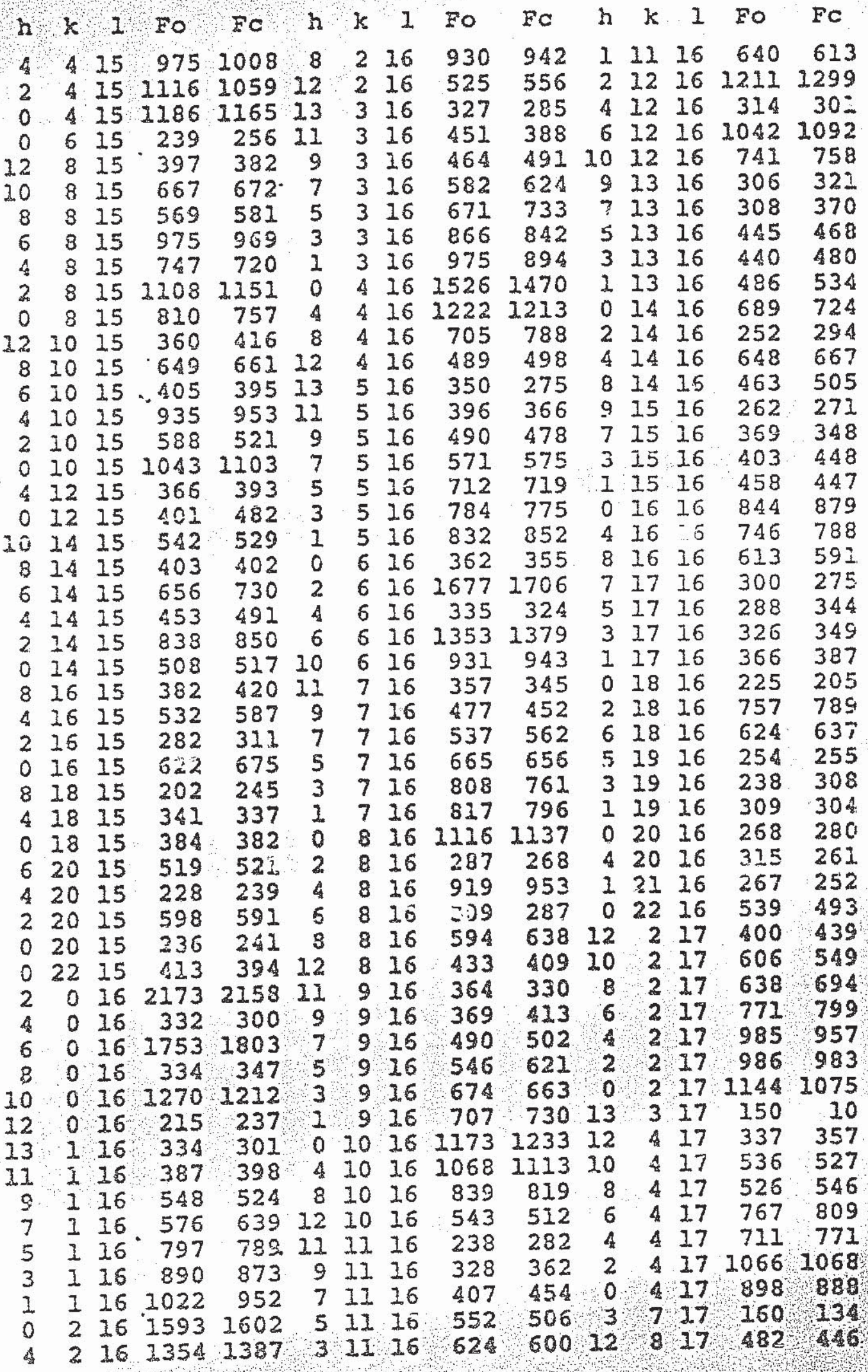


h K I Fo FC h K 1 FO FC h K I Fo FC

$\begin{array}{llllllllllllllllllllllll}10 & 8 & 17 & 488 & 493 & 3 & 7 & 18 & 207 & 199 & 8 & 10 & 19 & 366 & 406\end{array}$ $8 \quad 817 \quad 630 \quad 684 \quad 1 \quad 718 \quad 197 \quad 274 \quad 6 \quad 1019 \quad 469 \quad 520$

$\begin{array}{lllllllllllllllll}6 & 87 & 633 & 686 & 0 & 8 & 18 & 380 & 380 & 4 & 10 & 19 & 485 & 506\end{array}$

$\begin{array}{lllllllllllllllllllll}4 & 8 & 17 & 826 & 855 & 2 & 8 & 18 & 1235 & 1282 & 2 & 10 & 19 & 554 & 600\end{array}$

$\begin{array}{lllllllllllllll}2 & 8 & 17 & 754 & 778 & 4 & 8 & 18 & 410 & 385 & 0 & 10 & 19 & 541 & 537\end{array}$

$\begin{array}{lllllllllllllll}0 & 8 & 17 & 863 & 901 & 6 & 8 & 18 & 927 & 957 & 3 & 13 & 19 & 206 & 193\end{array}$

$\begin{array}{lllllllllllllll}10 & 10 & 17 & 357 & 395 & 8 & 8 & 18 & 365 & 323 & 8 & 14 & 19 & 473 & 469\end{array}$

$\begin{array}{lllllllllllllllll}8 & 10 & 17 & 407 & 434 & 10 & 8 & 18 & 574 & 616 & 6 & 14 & 19 & 335 & 395\end{array}$

$\begin{array}{lllllllllllllll}6 & 10 & 17 & 517 & 574 & 1 & 9 & 18 & 218 & 209 & 4 & 14 & 19 & 577 & 634\end{array}$

$\begin{array}{llllllllllllllll}4 & 10 & 17 & 536 & 600 & 0 & 10 & 18 & 269 & 303 & 2 & 14 & 19 & 436 & 498\end{array}$

$21017 \quad 693 \quad 710 \quad 2 \quad 10 \quad 18 \quad 1122 \quad 1179 \quad 0 \quad 1419 \quad 647 \quad 715$

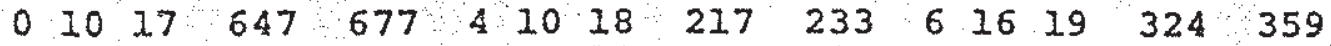

$21217 \quad 265 \quad 306 \quad 6 \quad 1018 \quad 9591002 \quad 4 \quad 16 \quad 19 \quad 218 \quad 271$

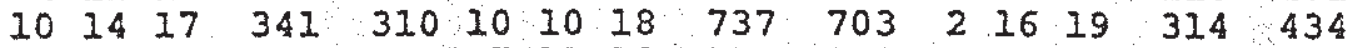

$8.1417571 \quad 554 \quad 7 \quad 11$ 18 $173 \quad 109$ o 16 19 $252 \quad 301$

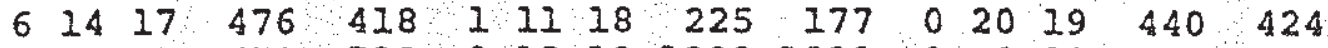

$41417 \quad 672 \quad 722 \quad 01218 \quad 12021329 \quad 0 \quad 0 \quad 20 \quad 887 \quad 839$

$21417 \quad 490 \quad 493 \quad 4 \quad 12 \quad 18 \quad 1155 \quad 1235 \quad 2 \quad 0 \quad 20 \quad 1289 \quad 1272$

$\begin{array}{lllllllllllllll}0 & 14 & 17 & 754 & 794 & 8 & 12 & 18 & 892 & 963 & 4 & 0 & 20 & 766 & 725\end{array}$

$616 \quad 17 \quad 457 \quad 417 \quad 0 \quad 1418 \quad 477 \quad 484 \quad 6 \quad 0 \quad 20 \quad 1032 \quad 994$

$\begin{array}{lllllllllllllllllllllll}46 & 17 & 355 & 287 & 2 & 14 & 18 & 662 & 710 & 8 & 0 & 20 & 501 & 502\end{array}$

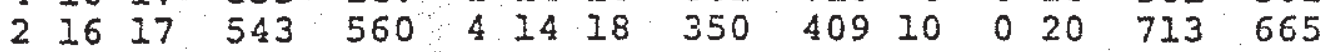

$\begin{array}{lllllllllllllllll}0 & 16 & 17 & 315 & 349 & 6 & 14 & 18 & 579 & 616 & 12 & 0 & 20 & 364 & 320\end{array}$

$\begin{array}{llllllllllllllll}6 & 10 & 17 & 265 & 195 & 8 & 14 & 18 & 238 & 273 & 11 & 1 & 20 & 448 & 432\end{array}$

$\begin{array}{lllllllllllllll}4 & 20 & 17 & 432 & 446 & 2 & 16 & 18 & 834 & 910 & 9 & 120 & 536 & 536\end{array}$

$\begin{array}{lllllllllllllll}2 & 20 & 17 & 325 & 285 & 6 & 16 & 18 & 709 & 729 & 7 & 1 & 20 & 740 & 707\end{array}$

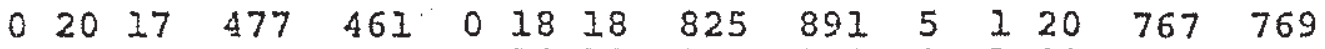

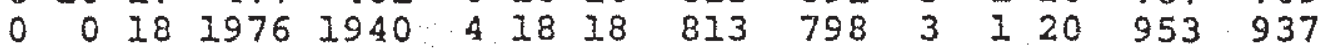

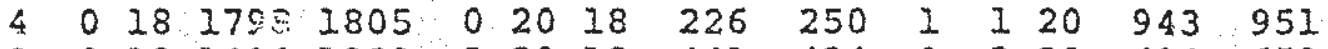

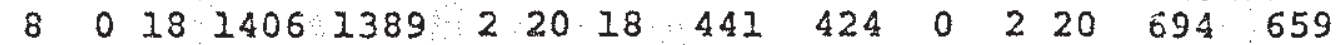

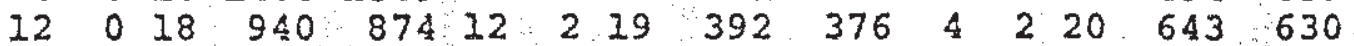

I $118 \quad 192 \quad 19510 \quad 2 \quad 19 \quad 512 \quad 512 \quad 8 \quad 2 \quad 20 \quad 494 \quad 500$

$\begin{array}{lllllllllllllllllll}0 & 2 & 28 & 670 & 644 & 8 & 2 & 19 & 573 & 575 & 10 & 2 & 20 & 196 & 176\end{array}$

$\begin{array}{llllllllllllllll}2 & 2 & 18 & 1464 & 1398 & 6 & 2 & 19 & 712 & 755 & 12 & 2 & 20 & 398 & 319\end{array}$

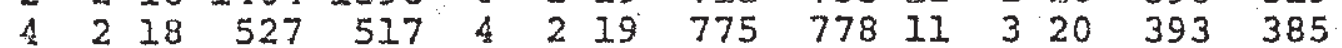

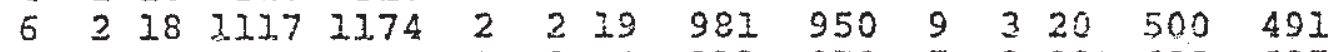

$\begin{array}{lllllllllllllll}10 & 2 & 18 & 817 & 799 & 0 & 2 & 19 & 911 & 872 & 7 & 3 & 20 & 633 & 637\end{array}$

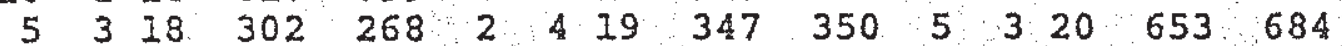

1 3 $18 \quad 267 \quad 271 \quad 10 \quad 419 \quad 464 \quad 464 \quad 3 \quad 320 \quad 839 \quad 843$

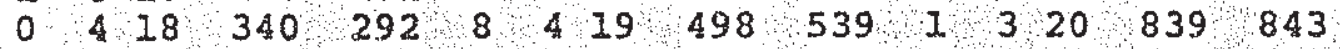

$241817661755 \quad 6 \$ 419 \quad 670,66900420 \% 673 \quad 651$

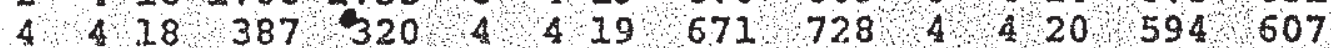

$641812681298 \quad 2 \quad 419 \quad 808 \quad 824 \quad 6 \quad 420$ L 47 214

$10418 \quad 832 \quad 829 \quad 0 \quad 419 \quad 845 \quad 813 \quad 8 \quad 4 \quad 20$ 433 478

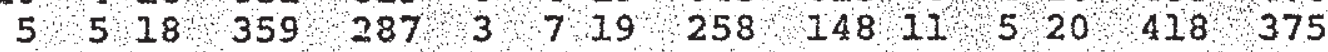

$\begin{array}{llllllllllllllll}3 & 5 & 18 & 233 & 230 & 10 & 8 & 19 & 382 & 415 & 9 & 5 & 20 & 439 & 460\end{array}$

1. $518 \quad 330 \quad 310 \quad 8 \quad 8 \quad 19 \quad 520 \quad 594 \quad 7 \quad 5 \quad 20 \quad 595 \quad 588$

$0 \quad 618 \quad 20482112 \quad 6 \quad 8 \quad 19 \quad 558 \quad 591 \quad 5 \quad 520 \quad 617$ 653

$\begin{array}{llllllllllllll}4 & 6 & 18 & 1731 & 1838 & 4 & 8 & 19 & 724 & 792 & 3 & 5 & 20 & 739\end{array}$

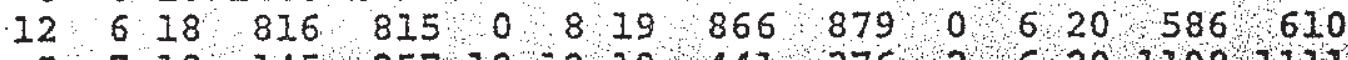

$5718,145 \quad 25710 \quad 1019 \quad 441$ 376 2 6 20 108 1111 


\begin{tabular}{|c|c|c|c|c|c|c|c|c|c|c|c|}
\hline 1 & Fo & $\mathrm{FC}$ & 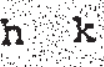 & 1 & F FO & $\mathrm{FC}$ & $\mathrm{h}$ & $\mathrm{k}$ & & & \\
\hline 20 & $\begin{array}{l}540 \\
879\end{array}$ & 531 & $\begin{array}{l}319 \\
119\end{array}$ & $\begin{array}{l}20 \\
20\end{array}$ & $\begin{array}{l}327 \\
302\end{array}$ & $\begin{array}{l}314 \\
315\end{array}$ & $\begin{array}{l}7 \\
5\end{array}$ & $\begin{array}{l}322 \\
3 \quad 22\end{array}$ & $\begin{array}{l}2 \\
2\end{array}$ & $\begin{array}{l}471 \\
460\end{array}$ & $\begin{array}{l}421 \\
451\end{array}$ \\
\hline 20 & 434 & 385 & $\frac{1}{0} \quad 2$ & 21 & 547 & 462 & 3 & 32 & & 540 & \\
\hline 20 & 620 & 583 & 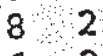 & 21 & 471 & 495 & & $\begin{array}{l}32 \\
42\end{array}$ & 2 & & \\
\hline 20 & 391 & 371 & 6 & $\begin{array}{l}21 \\
21\end{array}$ & $\begin{array}{l}634 \\
678\end{array}$ & $\begin{array}{l}676 \\
663\end{array}$ & $\begin{array}{l}0 \\
4\end{array}$ & $\begin{array}{l}42 \\
42\end{array}$ & 22 & $\begin{array}{l}960 \\
923\end{array}$ & 3 \\
\hline $\begin{array}{l}20 \\
20\end{array}$ & $\begin{array}{l}441 \\
600\end{array}$ & $\begin{array}{l}455^{\circ} \\
598\end{array}$ & $\begin{array}{ll}4 & 2 \\
2 & 2\end{array}$ & $\begin{array}{l}21 \\
21\end{array}$ & $\begin{array}{l}678 \\
853\end{array}$ & $\begin{array}{l}663 \\
844\end{array}$ & 8 & 42 & 2 & 765 & $\begin{array}{l}901 \\
728\end{array}$ \\
\hline 20 & 593 & 629 & 0 & 21 & 759 & 740 & 9 & 52 & 22 & 344 & 308 \\
\hline 20 & 770 & 77710 & 0 & 21 & 384 & 368 & 7 & 52 & 22 & 392 & 397. \\
\hline 20 & 787 & 771 & 8 & 21 & 55 & 564 & $\begin{array}{l}5 \\
3\end{array}$ & 52 & 22 & 519 & 421 \\
\hline 20 & 46 & $501:$ & 6 & 21 & $\begin{array}{l}466 \\
779\end{array}$ & 501 & $\begin{array}{l}3 \\
1\end{array}$ & $\begin{array}{l}52 \\
52\end{array}$ & $\begin{array}{l}22 \\
22\end{array}$ & 518 & 514 \\
\hline 20 & 40 & 461 & 4 & $\begin{array}{l}21 \\
21\end{array}$ & $\begin{array}{l}719 \\
535\end{array}$ & $\begin{array}{l}731 \\
572\end{array}$ & 0 & 62 & 22 & $\begin{array}{l}588 \\
194\end{array}$ & $\begin{array}{l}512 \\
248\end{array}$ \\
\hline $\begin{array}{l}20 \\
20\end{array}$ & 367 & $\begin{array}{l}359 \\
436\end{array}$ & $\begin{array}{l}2 \\
0\end{array}$ & $\begin{array}{l}21 \\
21\end{array}$ & $\begin{array}{l}535 \\
798\end{array}$ & .796 & 2 & 62 & 221 & 1323 & 1299 \\
\hline $\begin{array}{l}20 \\
20\end{array}$ & $\begin{array}{l}402 \\
503\end{array}$ & $\begin{array}{l}436 \\
5241\end{array}$ & $\begin{array}{l}0 \\
0\end{array}$ & 21 & 436 & 442 & 4 & 62 & 22 & 232 & 235 \\
\hline 20 & 520 & 622 & 8 & 21 & 379 & 393 & 6 & 62 & 221 & 1029 & 1065 \\
\hline 20 & 737 & 679 & 6 & 21 & 560 & $\begin{array}{l}64511 \\
550\end{array}$ & $\begin{array}{r}10 \\
9\end{array}$ & $6 ?$ & 22 & 740 & $\begin{array}{r}733 \\
270\end{array}$ \\
\hline 20 & 655 & 726 & 4 & 21 & 558 & $\begin{array}{l}559 \\
827\end{array}$ & $\begin{array}{l}9 \\
7\end{array}$ & $\begin{array}{l}7 \\
7\end{array}$ & 22 & $\begin{array}{l}285 \\
345\end{array}$ & $\begin{array}{l}279 \\
341\end{array}$ \\
\hline 20 & 619 & 636 & 2 & 21 & $\begin{array}{l}806 \\
600\end{array}$ & $\begin{array}{l}827 \\
645\end{array}$ & 5 & 7 & $\begin{array}{l}22 \\
22\end{array}$ & $\begin{array}{l}345 \\
408\end{array}$ & $\begin{array}{l}341 \\
389\end{array}$ \\
\hline 20 & 348 & 322 & 0 & 21 & $\begin{array}{l}6 \\
3\end{array}$ & 257 & 3 & 7 & 22 & 418 & 40 \\
\hline 20 & 568 & 5901 & 010 & $\begin{array}{l}21 \\
21\end{array}$ & 4 & 461 & $I$ & 7 & 22 & 516 & 458 \\
\hline 20 & 31 & 285 & $\begin{array}{lll}8 & 10 \\
6 & 10\end{array}$ & $\begin{array}{l}21 \\
21\end{array}$ & 35 & 344 & 0 & 8 & 22 & 764 & 757 \\
\hline 20 & 41. & $\begin{array}{l}459 \\
208\end{array}$ & $\begin{array}{ll}6 & 10 \\
4 & 10\end{array}$ & $0 \quad 21$ & 5 & 567 & 4 & 8 & 22 & 715 & 717 \\
\hline $\begin{array}{l}20 \\
20\end{array}$ & $\begin{array}{l}249 \\
373\end{array}$ & $\begin{array}{l}208 \\
368\end{array}$ & $\begin{array}{ll}4 & 1 \\
2 & 1\end{array}$ & $02 I$ & 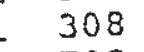 & 384 & 8 & 8 & 22 & 611 & $5^{\circ}$ \\
\hline 20 & 454 & 474 & 01 & $\begin{array}{ll}0 & 21\end{array}$ & . & 602 & 7 & 9 & 22 & $\begin{array}{l}358 \\
205\end{array}$ & $\begin{array}{l}326 \\
336\end{array}$ \\
\hline 20 & 426 & 501 & 11 & 121 & 187 & 53 & 5 & $\begin{array}{l}9 \\
9\end{array}$ & $\begin{array}{l}22 \\
22\end{array}$ & $\begin{array}{l}295 \\
358\end{array}$ & $\begin{array}{l}336 \\
416\end{array}$ \\
\hline 20 & 640 & 613 & 81 & $\begin{array}{ll}4 & 21\end{array}$ & 27 & $\begin{array}{l}255 \\
550\end{array}$ & $\begin{array}{l}3 \\
1\end{array}$ & $\begin{array}{l}9 \\
9\end{array}$ & $\begin{array}{l}22 \\
22\end{array}$ & $\begin{array}{l}358 \\
453\end{array}$ & $\begin{array}{l}410 \\
411\end{array}$ \\
\hline 20 & 590 & $6: 0$ & 61 & 421 & . & $\begin{array}{l}550 \\
344\end{array}$ & 0 & 10 & 22 & 790 & 860 \\
\hline 20 & 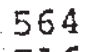 & 571 & 41 & $\begin{array}{ll}421 \\
0\end{array}$ & 651 & 684 & 41 & 10 & 22 & 724 & 769 \\
\hline 20 & 7 & 758 & 21 & $\begin{array}{lll}4 & 21 \\
4 & 21\end{array}$ & 392 & 390 & 8 & 10 & 22 & 572 & 577 \\
\hline 20 & 494 & 516 & 0 I & $\begin{array}{ll}4 & 21 \\
6 & 21\end{array}$ & 403 & 436 & 9 & 11 & 22 & 282 & 246 \\
\hline 20 & 5 & 629 & 4 & $\begin{array}{ll}6 & 21 \\
6 & 21\end{array}$ & 37 & 454 & 7 & 11 & 22 & 245 & 2 \\
\hline 20 & 35 & 392 & 01 & $\begin{array}{ll}6 & 21 \\
8 & 21\end{array}$ & 300 & 257 & 5 & 11 & 22 & 338 & \\
\hline 20 & 375 & 338 & $\begin{array}{lll}0 & 1 \\
0 & \end{array}$ & $\begin{array}{ll}8 & 21 \\
0 & 22\end{array}$ & 342 & 33 & 3 & 11 & 22 & 360 & 372 \\
\hline 20 & 341 & 401 & 2 & $\begin{array}{ll}0 & 22 \\
0 & 22\end{array}$ & $\therefore 1435$ & 1413 & 1 & 11 & 22 & 352 & 2 \\
\hline 20 & $51:$ & 489 & 2 & 022 & 184 & 245 & 0 & 12 & 22 & 161 & 8 \\
\hline 20 & 50 & $\begin{array}{l}512 \\
560\end{array}$ & 6 & 022 & 21121 & 1078 & 2 & 12 & 22 & 863 & 5 \\
\hline 20 & $\begin{array}{l}498 \\
375\end{array}$ & $\begin{array}{l}560 \\
308\end{array}$ & 10 & 022 & 752 & 723 & 6 & 12 & 22 & 698 & 5 \\
\hline 20 & 283 & 284 & 11 & 12 & 1 & 255 & 3 & 13 & 22 & $\begin{array}{l}338 \\
296\end{array}$ & \\
\hline 20 & 426 & 386 & 9 & 12 & 4 & 322 & 1 & & $\begin{array}{l}22 \\
22\end{array}$ & $\begin{array}{r}296 \\
434\end{array}$ & 4 \\
\hline 20 & 334 & 422 & 7 & 12 & 2 & 6 & 0 & $\begin{array}{l}14 \\
14\end{array}$ & 22 & $\begin{array}{l}434 \\
398\end{array}$ & 3 \\
\hline 20 & 4 & 497 & 5 & 12 & $\begin{array}{l}442 \\
535\end{array}$ & $\begin{array}{l}449 \\
548\end{array}$ & 4 & 14 & 22 & $\begin{array}{l}39 \\
17\end{array}$ & 0 \\
\hline 20 & 4 & 502 & 3 & 2 & $\begin{array}{l}535 \\
574\end{array}$ & 55 & 5 & 15 & 22 & & 2 \\
\hline 20 & 39 & 479 & 1 & $\begin{array}{l}12 \\
22\end{array}$ & $\begin{array}{l}574 \\
917\end{array}$ & 908 & 3 & 15 & 22 & & 2 \\
\hline 20 & 41 & 433 & 0 & $\begin{array}{ll}2 & 2 \\
2 & 2\end{array}$ & 274 & 212 & 1 & 15 & 22 & 25 & \\
\hline 20 & $\begin{array}{l}32 \\
37\end{array}$ & 384. & 2 & 22 & 834 & 817 & 0 & 16 & 22 & 552 & \\
\hline $\begin{array}{l}20 \\
20\end{array}$ & 37 & $\begin{array}{l}379 \\
275\end{array}$ & $\frac{4}{8}$ & 22 & 647 & & 4 & 16 & 22 & & 570 \\
\hline $\begin{array}{l}20 \\
20\end{array}$ & $\begin{array}{l}334 \\
380\end{array}$ & $\begin{array}{l}425 \\
454\end{array}$ & 11 & 32 & 285 & & 2 & 18 & 22 & 6 & 612 \\
\hline 620 & & 285 & $2=$ & 2 & 22 & & 10 & 2 & 23 & & \\
\hline
\end{tabular}




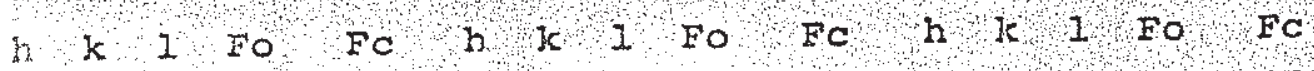

8. $223 \quad 46145461024 \quad 593 \quad 709 \quad 0 \quad 626 \quad 450 \quad 479$

6 $223 \quad 568 \quad 569 \quad 0 \quad 12 \quad 24 \quad 932 \quad 973 \quad 2 \quad 6 \quad 26 \quad 625 \quad 613$

$\begin{array}{lllllllllllll}2 & 23 & 527 & 592 \quad 4 \quad 12 & 24 & 858 & 870 \quad 4 & 6 & 26 & 411 & 453\end{array}$

$2 \quad 2.23 \quad 718 \quad 693 \quad 0 \quad 1424 \quad 235 \quad 214 \quad 6 \quad 6 \quad 26 \quad 501 \quad 538$

$\begin{array}{llllllllllllllllllllll}0 & 23 & 671 & 650 & 2 & 14 & 24 & 482 & 515 & 8 & 6 & 26 & 401 & 369\end{array}$

$10 \quad 4 \quad 23 \quad 417 \quad 378^{\circ} \quad 4 \quad 1424 \quad 233 \quad 208 \quad 7 \quad 726 \quad 393 \quad 374$

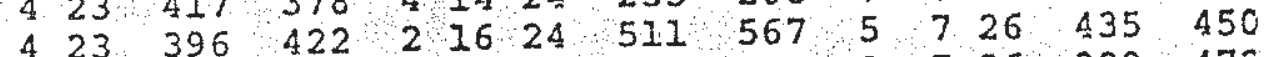

$423 \quad 500 \quad 520 \quad 8 \quad 2 \quad 25 \quad 484 \quad 447 \quad 3 \quad 7 \quad 26 \quad 389 \quad 472$

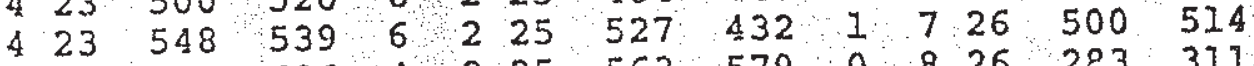

$\begin{array}{llllllllllllllll}4 & 23 & 654 & 606 & 4 & 2 & 25 & 563 & 579 & 0 & 8 & 26 & 293 & 311\end{array}$

$\begin{array}{llllllllllllll}4 & 23 & 607 & 581 & 2 & 2 & 25 & 535 & 504 & 7 & 9 & 26 & 369 & 355\end{array}$

$\begin{array}{lllllllllllllllllll}8 & 23 & 492 & 461 & 0 & 2 & 25 & 638 & 633 & 5 & 9 & 26 & 390 & 404\end{array}$

$\begin{array}{llllllllllllllllllll}8 & 23 & 393 & 434 & 8 & 4 & 25 & 421 & 349 & 3 & 9 & 26 & 425 & 453\end{array}$

$\begin{array}{lllllllllllllll}8 & 23 & 635 & 630 & 6 & 4 & 25 & 471 & 461 & 1 & 9 & 26 & 502 & 469\end{array}$

$\begin{array}{llllllllllllllll}8 & 23 & 517 & 549 & 4 & 4 & 25 & 479 & 454 & 0 & 10 & 26 & 369 & 395\end{array}$

$\begin{array}{llllllllllllllllll}8 & 23 & 739 & 715 & 2 & 4 & 25 & 612 & 561 & 4 & 10 & 26 & 386 & 355\end{array}$

$\begin{array}{llllllllllllllll}10 & 23 & 283 & 286 & 0 & 4 & 25 & 493 & 500 & 5 & 11 & 26 & 367 & 373\end{array}$

$\begin{array}{lllllllllllllll}10 & 23 & 445 & 420 & 8 & 8 & 25 & 422 & 398 & 3 & 11 & 26 & 324 & 384\end{array}$

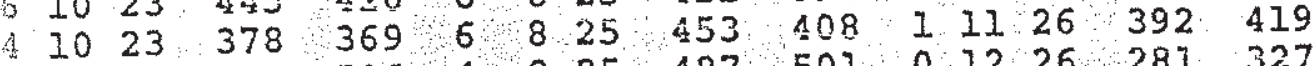

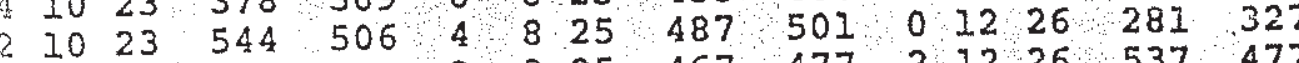

$\begin{array}{lllllllllllllll}0 & 10 & 23 & 382 & 405 & 2 & 8 & 25 & 467 & 477 & 2 & 12 & 26 & 537 & 477\end{array}$

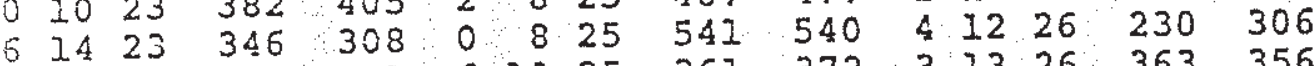

$414 \quad 23 \quad 461 \quad 501 \quad 6 \quad 1025 \quad 361 \quad 372 \quad 3 \quad 13 \quad 26 \quad 363 \quad 356$

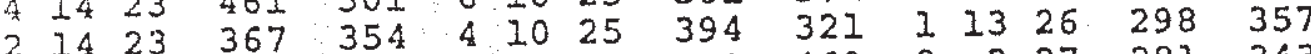

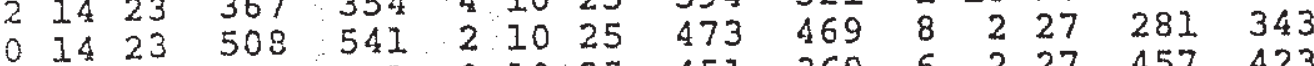

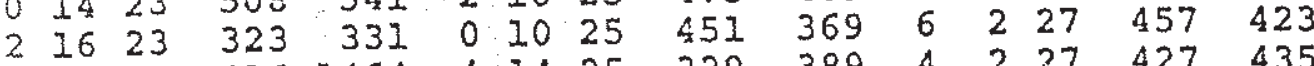

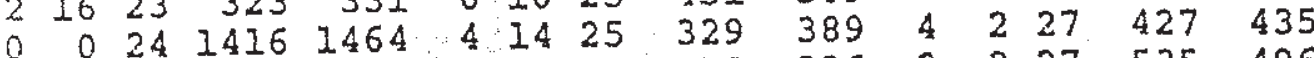

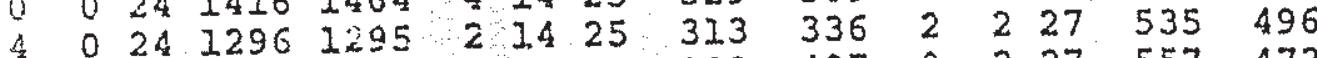

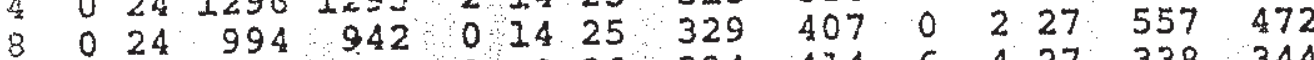

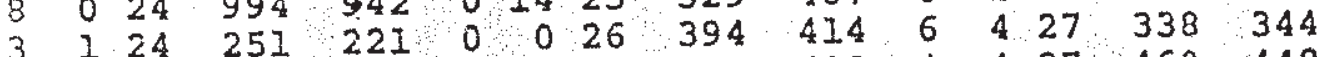

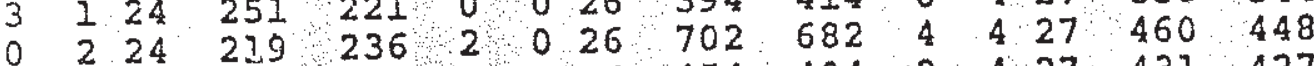

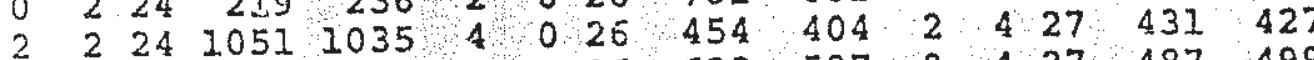

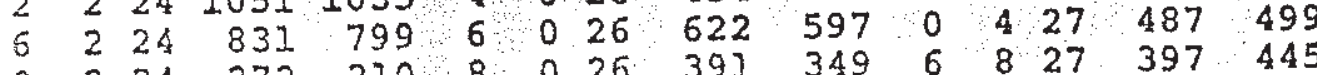

$\begin{array}{lllllllllllllll}8 & 2 & 24 & 272 & 210 & 8 & 0 & 26 & 391 & 349 & 6 & 8 & 27 & 397 & 45 \\ 7 & 1 & 26 & 413 & 421 & 4 & 8 & 27 & 316 & 326\end{array}$

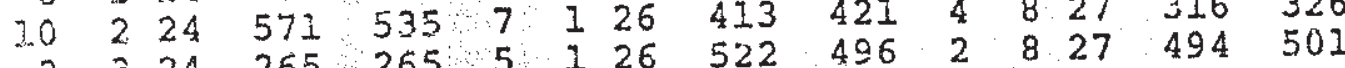

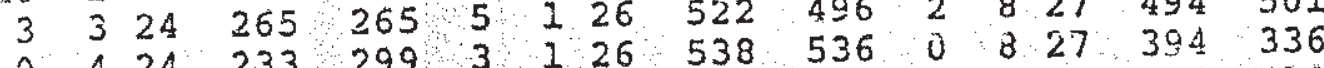

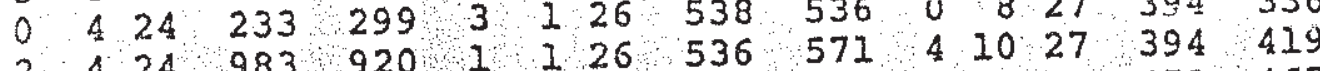

$\begin{array}{lllllllllllll}2 & 424 & 983 & 920 & 1 & 1 & 26 \\ 4 & 24 & 536 & 571 & 4 & 10 & 27 & 36\end{array}$

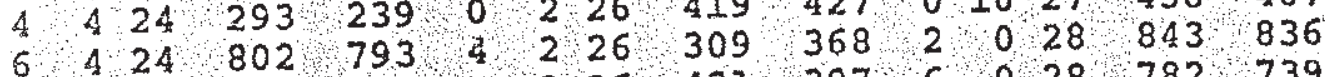

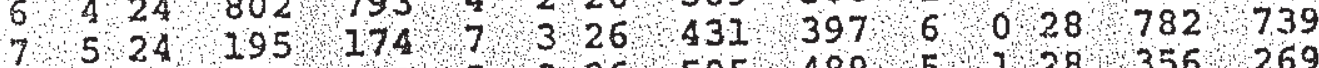

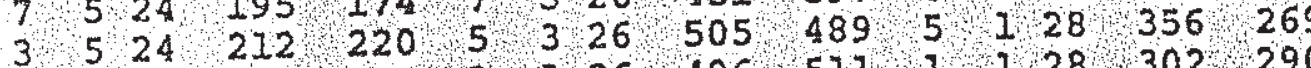

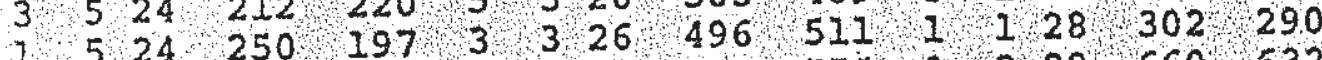

0.62412601167 I $326 \quad 511 \quad 556 \quad 0 \quad 2 \quad 28 \quad 660$ 632

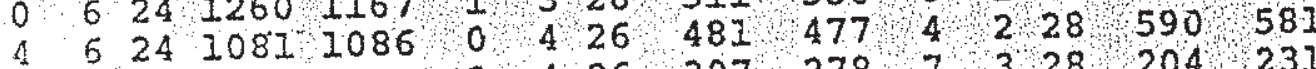

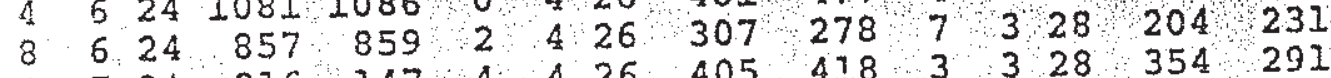

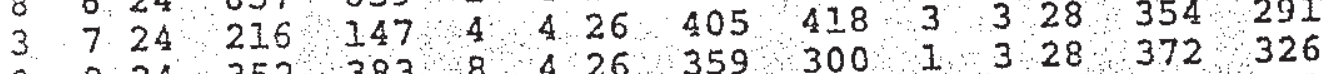

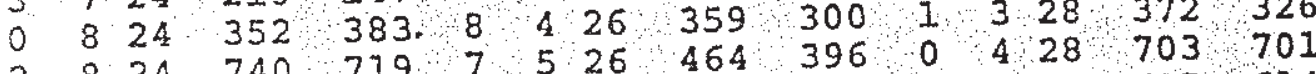

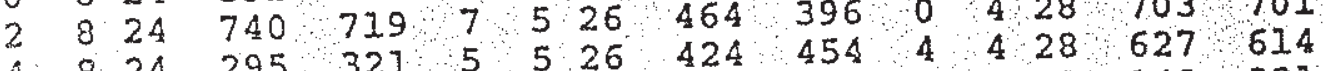

$\begin{array}{lllllllllllllll}4 & 8 & 24 & 295 & 321 & 5 & 5 & 26 & 424 & 454 & 4 & 4 & 28 & \\ 6 & 8 & 24 & 623 & 624 & 3 & 5 & 26 & 477 & 506 & 1 & 5 & 28 & 248 & 321\end{array}$

$21024 \quad 811 \quad 893 \quad 1 \quad 526 \quad 586 \quad 529 / 2,628 \quad 780 \quad 795$ 


$\begin{array}{rrrrr}\text { h K } & 1 & \text { FO } & \text { EC } \\ 6 & 6 & 28 & 630 & 662 \\ 1 & 7 & 28 & 164 & 266 \\ 0 & 8 & 28 & 474 & 477 \\ 4 & 8 & 28 & 417 & 420 \\ 3 & 9 & 28 & 269 & 218 \\ 0 & 10 & 28 & 592 & 656 \\ 4 & 10 & 28 & 524 & 517 \\ 6 & 2 & 29 & 302 & 311 \\ 4 & 2 & 29 & 473 & 429 \\ 2 & 2 & 29 & 385 & 366 \\ 0 & 2 & 29 & 422 & 468 \\ 4 & 4 & 29 & 306 & 331 \\ 2 & 4 & 29 & 425 & 405 \\ 0 & 4 & 29 & 349 & 363 \\ 4 & 8 & 29 & 339 & 384 \\ 2 & 8 & 29 & 337 & 332 \\ 0 & 8 & 29 & 477 & 410 \\ 2 & 10 & 29 & 338 & 349 \\ 0 & 10 & 29 & 338 & 349 \\ 4 & 0 & 30 & 708 & 746 \\ 2 & 2 & 30 & 524 & 532 \\ 2 & 4 & 30 & 608 & 610 \\ 0 & 6 & 30 & 801 & 795 \\ 2 & 2 & 31 & 336 & 346 \\ 0 & 2 & 31 & 381 & 365 \\ 2 & 4 & 31 & 330 & 316 \\ 0 & 4 & 31 & 292 & 313\end{array}$

